\title{
THE MEASURE OF THE SET OF ADMISSIBLE LATTICES
}

\section{WOLFGANG SCHMIDT}

Introduction. Let $S$ be a Borel set in $n$-dimensional space which does not contain the origin 0 . We assume that there is no $X$ so that both $X \in S$ and $-X \in S$. We say a point lattice $\Lambda$ is $S$-admissible, if there is no lattice point of $\Lambda$ in $S$. We denote by $A(S)$ the set of $S$ admissible lattices and by $V=V(S)$ the measure of $S$.

The main result of this paper is

THEOREM 4. If

$$
V \leqq n-1 \text { and } n \geqq 13 \text {, }
$$

then

$$
m(A(S))=\int_{\Omega \Lambda_{0} \in A(S) ; \Omega \in F} d \mu(\Omega)=e^{-V}(1-R),
$$

where

$$
|R|<6(3 / 4)^{n / 2} e^{4 V}+V^{n-1} n^{-n+1} e^{V+n} .
$$

Here $\Omega$ denotes a linear transformation of determinant $1, F$ is a fundamental region with respect to the subgroup of unimodular transformations of determinant 1 , and $\mu(\Omega)$ is the invariant measure on the space of linear transformations with determinant 1 , defined by C. L. Siegel [5], normalized so that

$$
\int_{F} d \mu(\Omega)=1
$$

$\Lambda_{0}$ denotes the lattice of points with integral coordinates.

Theorem 4 will be used to prove Theorem 5 which is an improvement of the Minkowski-Hlawka Theorem. We also prove two existence theorems which are in a certain sense converses of the MinkowskiHlawka Theorem (Theorem 6 and Theorem 7).

The author is very indebted to the referee who pointed out some errors of the originally submitted paper and made useful suggestions. The originally stated Theorem 1 was wrong.

Presented to the Society, June 15, 1957; received by the editors November 23, 1956 and, in revised form, June 3, 1957 and October 31, 1957. 
1. We define the lattice function

$$
\alpha(\Lambda)= \begin{cases}1, & \text { for } \Lambda \in A(S), \\ 0, & \text { for } \Lambda \notin A(S),\end{cases}
$$

and $\rho(\Lambda)$ to be the number of lattice points of $\Lambda$ in $S$. The usual bound for $\alpha(\Lambda)$, used for the proof of the Minkowski-Hlawka Theorem, is

$$
\alpha(\Lambda) \geqq 1-\rho(\Lambda) .
$$

In $\S 1$ we shall replace (5) by a better bound.

We define for $0 \leqq j \leqq k \leqq n, k>0$,

$$
\stackrel{j}{\rho_{k}}(\Lambda)
$$

to be the number of $k$-tuples $\left(X_{1}, \cdots, X_{k}\right)$ of different lattice points $X_{i}$ of $\Lambda$ with $X_{1} \in S, \cdots, X_{k} \in S$ and $\operatorname{dim}\left(X_{1}, \cdots, X_{k}\right)=j$. (Here the order is immaterial, that is, we count $k$ points of a $k$-tuple $\left(X_{1}, \cdots, X_{k}\right)$ only once and not $k$ ! times.)

We further define $\tau_{k}(\Lambda)$ and $\pi_{k}(\Lambda)$ by

$$
\tau_{k}(\Lambda)=\left\{\begin{array}{c}
\rho_{k}^{k}(\Lambda), \text { if } k \text { is even, } \\
k \\
\rho_{k}(\Lambda)+{ }_{k}^{k-1}(\Lambda), \text { if } k \text { is odd }
\end{array}\right.
$$

and

$$
\pi_{k}(\Lambda)=\left\{\begin{array}{c}
\rho_{k}(\Lambda), \text { if } k \text { is odd, } \\
k \\
\rho_{k}(\Lambda)+\rho_{k}^{k-1}(\Lambda), \text { if } k \text { is even }
\end{array}\right.
$$

Since $0 \notin S, \tau_{1}(\Lambda)=\rho_{1}^{1}(\Lambda)+\rho_{1}^{0}(\Lambda)=\rho_{1}^{1}(\Lambda)=\rho(\Lambda)$.

The purpose of this section is to prove

THEOREM 1.

$$
1+\sum_{k=1}^{g}(-1)^{k} \pi_{k}(\Lambda) \geqq \alpha(\Lambda) \geqq 1+\sum_{k=1}^{h}(-1)^{k} \tau_{k}(\Lambda),
$$

for any odd $h \leqq n$ and any even $g \leqq n$.

For example, we have for $h=1$ and $h=3$

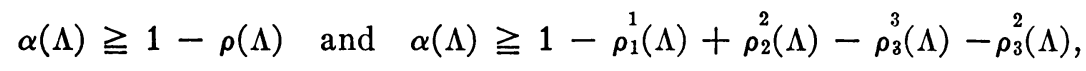

respectively. For the proof of Theorem 1 we need some lemmas. We consider the numbers

$$
A_{m}^{h}=\sum_{k=0}^{h}\left(\begin{array}{l}
m \\
k
\end{array}\right)(-1)^{k} \quad(0 \leqq h \leqq m, m>0) .
$$


LEMNA 1.

$$
\begin{aligned}
& A_{m}^{h} \leqq 0, \text { if } h \text { is odd; } \\
& A_{m}^{h} \geqq 0, \text { if } h \text { is even. }
\end{aligned}
$$

Proof of Lemma 1. We first assume $h<m / 2$. Then we have

$$
\left(\begin{array}{c}
m \\
r-1
\end{array}\right) \leqq\left(\begin{array}{c}
m \\
r
\end{array}\right)
$$

if $r \leqq h$. Therefore, if $h$ is odd, we see that

$$
A_{m}^{h}=-\sum\left[\begin{array}{c}
1 \leqq r \leqq h \\
r \text { odd }
\end{array}\right]\left\{\left(\begin{array}{c}
m \\
r
\end{array}\right)-\left(\begin{array}{c}
m \\
r-1
\end{array}\right)\right\} \leqq 0 ;
$$

and, if $h$ is even,

$$
A_{m}^{h}=1+\sum\left[\begin{array}{c}
1 \leqq r \leqq h \\
r \text { even }
\end{array}\right]\left\{\left(\begin{array}{c}
m \\
r
\end{array}\right)-\left(\begin{array}{c}
m \\
r-1
\end{array}\right)\right\} \geqq 0 .
$$

If $m>h \geqq m / 2$, then $m-(h+1)<m / 2$ and

$$
\begin{aligned}
A_{m}^{h} & =\sum_{k=0}^{h}\left(\begin{array}{c}
m \\
k
\end{array}\right)(-1)^{k}=\sum_{k=0}^{m}\left(\begin{array}{l}
m \\
k
\end{array}\right)(-1)^{k}-\sum_{k=h+1}^{m}\left(\begin{array}{l}
m \\
k
\end{array}\right)(-1)^{k} \\
& =0-\sum_{k=0}^{m-(h+1)}\left(\begin{array}{c}
m \\
k
\end{array}\right)(-1)^{m+k}=(-1)^{m+1} A_{m}^{m-(h+1)} .
\end{aligned}
$$

Thus, if $h$ is odd, we obtain the following:

If $m$ is even, then $A_{m}^{m-(h+1)} \geqq 0,(-1)^{m+1}=-1$, and so $A_{m}^{h} \leqq 0$;

if $m$ is odd, then $A_{m}^{m-(h+1)} \leqq 0,(-1)^{m+1}=1$, and so $A_{m}^{h} \leqq 0$.

In a similar way we can prove that, if $h$ is even, then $A_{m}^{h} \geqq 0$. If $m=h, A_{m}^{m}=0$.

Lemma 2. Let $a_{0}, a_{1}, a_{2}, \cdots, a_{m}$ be real non-negative numbers, for which

$$
1=a_{0}=a_{1}, \quad a_{2 t} \geqq a_{2 t+2} \quad(0 \leqq 2 t \leqq m-2)
$$

and

$$
a_{2 t} \leqq a_{2 t+1} \quad(0 \leqq 2 t \leqq m-1)
$$

hold. Then we have

$$
\sum_{k=0}^{h}\left(\begin{array}{c}
m \\
k
\end{array}\right)^{\prime}(-1)^{k} a_{k} \leqq \leqq 0
$$


if either $h$ is odd and $h \leqq m$, or if $h=m$.

But if $b_{0}, b_{1}, b_{2}, \cdots, b_{m}$, are real non-negative numbers, for which

$$
1=b_{0}=b_{1}, \quad b_{2 t-1} \geqq b_{2 t+1} \quad(2 \leqq 2 t \leqq m-1)
$$

and

$$
b_{2 t-1} \leqq b_{2 t}
$$

hold, then

$$
\sum_{k=0}^{o}\left(\begin{array}{l}
m \\
k
\end{array}\right)(-1)^{k} b_{k} \geqq 0,
$$

if either $g$ is even and $g \leqq m$, or if $g=m$.

Proof of Lemma 2. First we consider the case when (7a) and (8a) hold. We may assume that $a_{2 t+1}=a_{2 t}$. Then, using partial summation and Lemma 1, we have

$$
\begin{aligned}
\sum_{k=0}^{h}\left(\begin{array}{l}
m \\
k
\end{array}\right)(-1)^{k} a_{k}= & \sum\left[\begin{array}{c}
1 \leqq \\
t \text { odd }
\end{array}\right]\left(a_{t-1}-a_{t+1}\right) \sum_{k=0}^{t}\left(\begin{array}{l}
m \\
k
\end{array}\right)(-1)^{k} \\
& +a_{h} \sum_{k=0}^{h}\left(\begin{array}{l}
m \\
k
\end{array}\right)(-1)^{k} \\
& \leqq a_{h} \sum_{k=0}^{h}\left(\begin{array}{l}
m \\
k
\end{array}\right)(-1)^{k} .
\end{aligned}
$$

Now the right side is less than or equal to 0 , if $h$ is odd, or if $h=m$. So (9a) is true. Similarly (7b) and (8b) imply (9b).

Lemma 3. Let $\Lambda$ be a lattice with $\rho(\Lambda)=m>0$. We define numbers $a_{0}, a_{1}, a_{2}, \cdots, a_{m}$ and $b_{0}, b_{1}, b_{2}, \cdots, b_{m}$ by $a_{0}=b_{0}=1$ and

$$
\tau_{k}(\Lambda)=a_{k}\left(\begin{array}{l}
m \\
k
\end{array}\right) \quad \text { and } \quad \pi_{k}(\Lambda)=b_{k}\left(\begin{array}{l}
m \\
k
\end{array}\right) \quad(1 \leqq k \leqq m) .
$$

Now we assert the following: The $a_{k}$ satisfy (7a) and (8a), the $b_{k}$ satisfy (7b) and (8b).

Proof of Lemma 3. We have

$$
\tau_{1}(\Lambda)=m=a_{1}\left(\begin{array}{l}
m \\
1
\end{array}\right)=a_{1} m
$$

and therefore $a_{1}=1$. Defining constants $c_{k}$ by

$$
\stackrel{k}{\rho_{k}}(\Lambda)=c_{k}\left(\begin{array}{l}
m \\
k
\end{array}\right)
$$


we obtain

$$
\begin{aligned}
c_{k+1}\left(\begin{array}{c}
m \\
k+1
\end{array}\right)=\begin{array}{c}
k+1 \\
\rho_{k+1}
\end{array}(\Lambda) \\
\quad=\left\{\text { the number of }(k+1) \text {-tuples }\left(X_{1}, \cdots, X_{k+1}\right)\right. \text { of lattice points of } \\
\left.\quad \Lambda \text { with } X_{1} \in S, \cdots, X_{k+1} \in S \text { of dimension } k+1\right\} \\
\leqq \rho_{k}^{k}(\Lambda) \frac{m-k}{k+1}=c_{k}\left(\begin{array}{c}
m \\
k
\end{array}\right) \frac{m-k}{k+1}=c_{k}\left(\begin{array}{c}
m \\
k+1
\end{array}\right)
\end{aligned}
$$

The inequality holds because each $(k+1)$-tuple considered can be represented as the union of a $k$-tuple of linearly independent points of $\Lambda$ in $S$ and another point of $\Lambda$ in $S$ in $k+1$ ways. But there are $\rho_{k}^{k}(\Lambda)$ such $k$-tuples and a $k$-tuple given, there are $m-k$ other points of $\Lambda$ in $S$.

Dividing by

$$
\left(\begin{array}{c}
m \\
k+1
\end{array}\right)
$$

we obtain $c_{k+1} \leqq c_{k}$. Since, for even $k>0, a_{k}=c_{k}$, we have $a_{2 t} \geqq a_{2 t+2}$ for $t>0$. Also $a_{0}=a_{1}=c_{1} \geqq c_{2}=a_{2}$. Hence the $a_{k}$ satisfy (7a). If $t>0$, then

$$
\begin{aligned}
a_{2 t+1} & \left(\begin{array}{c}
m \\
2 t+1
\end{array}\right)=\tau_{2 t+1}(\Lambda)=\rho_{2 t+1}^{2 t+1}(\Lambda)+\rho_{2 t+1}^{2 t}(\Lambda) \\
= & \left\{\text { the number of }(2 t+1) \text {-tuples }\left(X_{1}, \cdots, X_{2 t+1}\right)\right. \text { of different lattice } \\
& \text { points of } \left.\Lambda \text { satisfying } X_{1} \in S, \cdots, X_{2 t+1} \in S \text { of dimension } \geqq 2 t\right\} \\
\geqq & \rho_{2 t}^{2 t}(\Lambda) \frac{m-2 t}{2 t+1}=\tau_{2 t}(\Lambda) \frac{m-2 t}{2 t+1}=a_{2 t}\left(\begin{array}{l}
m \\
2 t
\end{array}\right) \frac{m-2 t}{2 t+1}=a_{2 t}\left(\begin{array}{c}
m \\
2 t+1
\end{array}\right) .
\end{aligned}
$$

Dividing by

$$
\left(\begin{array}{c}
m \\
2 t+1
\end{array}\right)
$$

we obtain $a_{2 t+1} \geqq a_{2 t}$ and (8a).

If, in the above proof we replace $a_{k}$ by $b_{k}, \tau_{k}$ by $\pi_{k}$, even by odd, and in places $2 t+1$ by $2 t$, then we obtain $(7 \mathrm{~b})$ and $(8 \mathrm{~b})$.

Proof of Theorem 1. Again let $\Lambda$ be a lattice with $\rho(\Lambda)=m>0$. Let the numbers $a_{k}$ and $b_{k}$ be defined by (10). Then the $a_{k}$ satisfy (7a) and (8a), the $b_{k}$ satisfy (7b) and (8b). If therefore $h$ is odd, $h \leqq n, h \leqq m$, we have 


$$
1+\sum_{k=1}^{h}(-1)^{k} \tau_{k}(\Lambda)=\sum_{k=0}^{h}(-1)^{k}\left(\begin{array}{l}
m \\
k
\end{array}\right) a_{k} \leqq 0=\alpha(\Lambda),
$$

by Lemma 2 . But if $h \leqq n, h \geqq m$, we obtain the same result:

$$
1+\sum_{k=1}^{h}(-1)^{k} \tau_{k}(\Lambda)=\sum_{k=0}^{m}(-1)^{k}\left(\begin{array}{l}
m \\
k
\end{array}\right) a_{k} \leqq 0=\alpha(\Lambda) .
$$

In case $g$ is even, $g \leqq n, g \leqq m$, we have

$$
1+\sum_{k=1}^{g}(-1)^{k} \pi_{k}(\Lambda)=\sum_{k=0}^{g}(-1)^{k}\left(\begin{array}{l}
m \\
k
\end{array}\right) b_{k} \geqq 0=\alpha(\Lambda) ;
$$

and for $g \leqq n, g \geqq m$

$$
1+\sum_{k=1}^{g}(-1)^{k} \pi_{k}(\Lambda)=\sum_{k=0}^{m}(-1)^{k}\left(\begin{array}{l}
m \\
k
\end{array}\right) b_{k} \geqq 0=\alpha(\Lambda) .
$$

Therefore Theorem 1 is true if $\rho(\Lambda)>0$. It is evidently true if $\rho(\Lambda)=0$.

2. We now calculate the integrals of $\rho_{k}^{k}(\Lambda)$ and $\rho_{k}^{k-1}(\Lambda)$ over the space of lattices with determinant 1 .

Theorem 2. Suppose $k<n$. Then $\rho_{k}^{k}(\Lambda)$ is Borel-measurable in the space of lattices of determinant 1 and

$$
R_{k}^{k}=\int_{F}^{\substack{k \\ \rho_{k}}}\left(\Omega \Lambda_{0}\right) d \mu(\Omega)=\frac{1}{k !} V^{k} .
$$

Proof of Theorem 2. First, by the definition of $\rho_{k}^{j}(\Lambda)$, we see

$$
\rho_{k}^{j}(\Lambda)=\frac{1}{k !} \sum\left[\begin{array}{c}
X_{1} \in \Lambda, \cdots, X_{k} \in \Lambda \\
\operatorname{dim}\left(X_{1}, \cdots, X_{k}\right)=j \\
X_{i} \neq X_{h} \text {, if } i \neq h
\end{array}\right] \rho\left(X_{1}\right) \cdots \rho\left(X_{k}\right),
$$

where $\rho(X)$ is the characteristic function of $S$.

On the other hand, we observe the following theorem, stated by C. L. Siegel [5] and proved by C. A. Rogers ${ }^{1}$ [2]: If

then

$$
\psi(\Lambda)=\sum\left[\begin{array}{l}
X_{1} \in \Lambda, \cdots, X_{k} \in \Lambda \\
\operatorname{dim}\left(X_{1}, \cdots, X_{k}\right)=k
\end{array}\right] \rho\left(X_{1}\right) \cdots \rho\left(X_{k}\right),
$$

$$
\int_{F} \psi\left(\Omega \Lambda_{0}\right) d \mu(\Omega)
$$

${ }^{1}$ C. A. Rogers [2], Theorem 3, take $h=0$. 
exists and is equal to

$$
\int \cdots \int \rho\left(X_{1}\right) \cdots \rho\left(X_{k}\right) d X_{1} \cdots d X_{k}
$$

Theorem 2 is an immediate consequence of these two results.

Theorem 3. Suppose $k<n$. Then $\rho_{\boldsymbol{k}}^{k-1}(\Lambda)$ is Borel measurable in the space of lattices with determinant 1 , and

$$
\begin{aligned}
& R_{k}^{k-1}=\int_{F} \rho_{k}^{k-1}\left(\Omega \Lambda_{0}\right) d \mu(\Omega) \\
&=\frac{1}{k !} \sum_{l=1}^{k} \sum_{q=1}^{\infty} \sum_{D} \frac{1}{q^{n}} \int \cdots \int \rho\left(X_{1}\right) \cdots \\
& \\
& \quad \rho\left(X_{k-1}\right) \rho\left(\sum_{i=1}^{k-1} \frac{d_{i}}{q} X_{i}\right) d X_{1} \cdots d X_{k-1} .
\end{aligned}
$$

Moreover,

$$
R_{k}^{k-1} \leqq \frac{V^{k-1}}{(k-1) !}\left[3^{k}(3 / 4)^{n / 2}+5^{k} 2^{-n}\right] .
$$

The sum in (13) is over all integral vectors $D=\left(d_{1}, \cdots, d_{k-1}\right)$, which have highest common factor relative prime to $q$, and which obey $\left|d_{j}\right|<q$ for $j<l$ and $\left|d_{j}\right| \leqq q$ for $j \geqq l$. Further, if $q=1, D$ is not $(0,0, \cdots, 0)$ nor of the form $(0, \cdots, 0,1,0, \cdots, 0)$.

Before we can give a proof of Theorem 3 we need some lemmas.

LEMma 4.

$$
\begin{aligned}
& \sum\left[\begin{array}{c}
X_{1} \in \Lambda, \cdots, X_{k} \in \Lambda \\
\operatorname{dim}\left(X_{1}, \cdots, X_{k}\right)=k-1 \\
X_{i} \neq X_{j} \text { if } i \neq j
\end{array}\right] \rho\left(X_{1}\right) \cdots \rho\left(X_{k}\right) \\
& =\sum_{l=1}^{k} \sum_{q=1}^{\infty} \sum_{D} \sum\left[\begin{array}{c}
Y_{1} \in \Lambda, \cdots, Y_{k-1} \in \Lambda \\
\operatorname{dim}\left(Y_{1}, \cdots, Y_{k-1}\right)=k-1 \\
\sum_{i=1}^{k-1} d_{i} / q Y_{i} \in \Lambda
\end{array}\right] \rho\left(Y_{1}\right) \cdots \\
& \rho\left(Y_{k-1}\right) \rho\left(\sum_{i=1}^{k-1} \frac{d_{i}}{q} Y_{i}\right),
\end{aligned}
$$

where the sum on the right hand side is to be taken over the same set of vectors $D$ as in Theorem 3. 
Proof of Lemma 4. If $X_{1}, \cdots, X_{k}$ is in the sum of the left hand side of (15), then $\operatorname{dim}\left(X_{1}, \cdots, X_{k}\right)=k-1$. Hence, the vectors $X_{1}, \cdots, X_{k}$ span $a(k-1)$-dimensional space. In this space we construct a system of orthogonal unit vectors $e_{1}, e_{2}, \cdots, e_{k-1}$. We write $X_{j}$ in the form

$$
X_{j}=\sum_{i=1}^{k-1} a_{i j} e_{i}
$$

We define $A_{j}(1 \leqq j \leqq k)$ to be the determinant

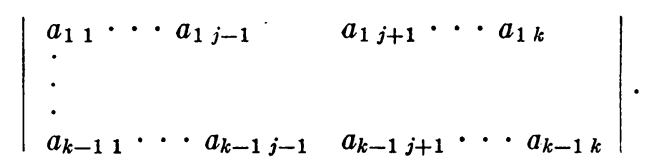

There exists a unique $l$, such that

$$
\left|A_{j}\right|<\left|A_{l}\right| \text {, if } j<l \text {, and }\left|A_{j}\right| \leqq\left|A_{l}\right| \text {, if } j \geqq l .
$$

This $k$-tuple $\left(X_{1}, \cdots, X_{k}\right)$ corresponds to the $(k-1)$-tuple $\left(Y_{1}, \cdots, Y_{k-1}\right)$, defined by

$$
\begin{aligned}
& Y_{1}=X_{1}, \cdots, Y_{l-1}=X_{l-1}, \\
& Y_{l}=X_{l+1}, \cdots, Y_{k-1}=X_{k},
\end{aligned}
$$

and to the number $l$, to the vector $D=\left(d_{1}, \cdots, d_{k-1}\right)$ and $q$, uniquely determined by

$$
X_{l}=\sum_{i=1}^{k-1} \frac{d_{i}}{q} Y_{i}
$$

and

$$
\text { g.c.d. }\left(d_{1}, \cdots, d_{k-1}, q\right)=1 \text {. }
$$

Because of our choice of $l$ to make $\left|A_{l}\right|$ maximal we have

$$
\left|d_{t}\right|<q \text {, if } t<l \text {, and }\left|d_{t}\right| \leqq q \text {, if } t \geqq l .
$$

If $q=1$, then $D\left(d_{1}, \cdots, d_{k-1}\right)$ is not of the form $(0,0, \cdots, 0)$ or $(0, \cdots, 0,1,0, \cdots, 0)$.

Since $l, d, q, Y_{j}$ do not depend on any particular choice of the unit vectors $e_{1}, \cdots, e_{k-1}$, there corresponds to each term on the left side of (15) exactly one term on the right hand side. If, conversely, there are $l, D, q, Y_{j}$ on the right side of (15), then we take the correspondence 


$$
\begin{gathered}
X_{1}=Y_{1}, \cdots, X_{l-1}=Y_{l-1}, \quad X_{l}=\sum_{i=1}^{k-1} \frac{d_{i}}{q} Y_{i}, \\
X_{l+1}=Y_{l}, \cdots, X_{k}=Y_{k-1} .
\end{gathered}
$$

These two mappings are one-one and inverse to each other. This proves the lemma.

Lemma 5 (C. A. Rogers). Let $\rho\left(X_{1}, \cdots, X_{m}\right)$ be a Borel measurable function which is integrable in the Lebesgue sense over the whole $\left(X_{1}, \cdots, X_{m}\right)$-space. Let $q$ be a positive integer and $D=\left(d_{1}, \cdots, d_{m}\right)$ be an integral vector with highest common factor relatively prime to $q$. Then the lattice function

$$
\omega(\Lambda)=\sum\left[\begin{array}{c}
X_{1} \in \Lambda, \cdots, X_{m} \in \Lambda \\
\operatorname{dim}\left(X_{1}, \cdots, X_{m}\right)=m \\
\sum_{i=1}^{m} d_{i} / q, X_{i} \in \Lambda
\end{array}\right] \rho\left(X_{1}, \cdots, X_{m}\right)
$$

is Borel measurable in the space of lattices of determinant 1, and

$$
\int_{F} \omega\left(\Omega \Lambda_{0}\right) d \mu(\Omega)=\frac{1}{q^{n}} \int \cdots \int \rho\left(X_{1}, \cdots, X_{m}\right) d X_{1} \cdots d X_{m} .
$$

Proof of Lemma 5. Lemma 5 is essentially the case $h=1$ of Theorem 3 of C. A. Rogers [2]. The only difference is that we write $1 / q$ instead of $e_{1} / q$ as in Rogers, where $e_{1}=$ g.c.d. $\left(\epsilon_{1}, q\right)$ and $\epsilon_{1}$ is the elementary divisor of the matrix $D$. But since g.c.d. $\left(d_{1}, \cdots, d_{m}, q\right)=1$, we have $e_{1}=$ g.c.d. $\left(\epsilon_{1}, q\right)=1$.

Lemma 6 (C. A. Rogers). If $\rho(X)$ is a characteristic function, then

$$
\iint \rho(X) \rho(Y) \rho(X+Y+a) d X d Y \leqq 2(3 / 4)^{n / 2}\left(\int \rho(X) d X\right)^{2} .
$$

Proof of Lemma 6. See C. A. Rogers [3, Lemma 5].

Proof of THEOREM 3. (13) is a straightforward consequence of (12), Lemma 4 and Lemma 5 (take $m=k-1$ ). Therefore only (14) remains to be proved. (14) implies that both sides of (13) are finite. We evidently have

$$
\begin{aligned}
R_{k}^{k-1} \leqq \frac{1}{k !} k \sum_{q=1}^{\infty} \sum_{D} \frac{1}{q^{n}} \int & \cdots \int \rho\left(X_{1}\right) \cdots \\
& \rho\left(X_{k-1}\right) \rho\left(\sum_{i=1}^{k-1} \frac{d_{i}}{q} X_{i}\right) d X_{1} \cdots d X_{k-1},
\end{aligned}
$$


but now the summation is to be taken over all integral $D$ with highest common factor relatively prime to $q$ and $\left|d_{j}\right| \leqq q$. If $q=1$, then $D \neq(0,0, \cdots, 0)$ and $\neq(0, \cdots, 0,1,0, \cdots, 0)$.

In (19) we mean that the inequality holds, if the right hand side is finite. We estimate the sum on the right hand side. We derive upper bounds (A) for the terms with $q=1$ and (B) for terms with $q>1$.

(A) There are $\leqq 3^{k-1}$ possibilities for $D$. $D$ either has two elements $d_{i}, d_{j}$, both different from zero, or $D$ is the form $(0, \cdots, 0,-1$, $0, \cdots, 0)$. In the first case we have, by Lemma 6 ,

$$
\begin{array}{r}
\int \cdots \int \rho\left(X_{1}\right) \cdots \rho\left(X_{k-1}\right) \rho\left( \pm X_{i_{1}} \pm X_{i_{2}} \pm \cdots\right) d X_{1} \cdots d X_{k-1} \\
\leqq 2(3 / 4)^{n / 2}\left(\int \rho(X) d X\right)^{k-1}=2(3 / 4)^{n / 2} V^{k-1}
\end{array}
$$

If $D$ is of the form $(0, \cdots, 0,-1,0, \cdots, 0)$, then

$$
\int \cdots \int \rho\left(X_{1}\right) \cdots \rho\left(X_{k-1}\right) \rho\left(-X_{i}\right) d X_{1} \cdots d X_{k-1}=0 .
$$

Thus

$$
\begin{array}{r}
\sum_{D} \frac{1}{1^{n}} \int \cdots \int \rho\left(X_{1}\right) \cdots \rho\left(X_{k-1}\right) \rho\left(\sum_{i=1}^{k-1} \frac{d_{i}}{q} X_{i}\right) d X_{1} \cdots d X_{k-1} \\
\leqq\left[3^{k}(3 / 4)^{n / 2}\right] V^{k-1}
\end{array}
$$

(B) For a fixed $q>1$ the number of vectors $D$ is at most $(2 q+1)^{k-1}$ $\leqq(5 / 2)^{k-1} q^{k-1}$. Consequently,

$$
\sum_{q=2}^{\infty} \sum_{D} \frac{1}{q^{n}} \int \cdots \int \rho\left(X_{1}\right) \cdots \rho\left(X_{k-1}\right) \rho\left(\sum_{i=1}^{k-1} \frac{d_{i}}{q} X_{i}\right) d X_{1} \cdots d X_{k-1}
$$

$$
\begin{aligned}
& \leqq(5 / 2)^{k-1} \sum_{q=2}^{\infty} q^{k-1-n} V^{k-1} \leqq(5 / 2)^{k-1} 2^{k+1-n} \sum_{q=2}^{\infty} \frac{1}{q^{2}} V^{k-1} \\
& <(5 / 2)^{k-1} 2^{k+1-n} V^{k-1}<5^{k} 2^{-n} V^{k-1} .
\end{aligned}
$$

By (19), (20) and (21) we get the upper bound

$$
R_{k}^{k-1} \leqq \frac{1}{(k-1) !}\left[3^{k}(3 / 4)^{n / 2}+5^{k} 2^{-n}\right] V^{k-1} .
$$

3. Proof of Theorem 4. Assume that (1) is satisfied. If $h$ is odd and $h<n$, we infer from Theorem 1 that 


$$
\begin{aligned}
\int_{F} \alpha\left(\Omega \Lambda_{0}\right) d \mu(\Omega) & \geqq 1+\sum_{k=1}^{h}(-1)^{k} \int_{F} \tau_{k}\left(\Omega \Lambda_{0}\right) d \mu(\Omega) \\
& \geqq 1+\sum_{k=1}^{h}(-1)^{k} R_{k}^{k}-\sum_{k=2}^{h} R_{k}^{k-1} \\
& \geqq 1+\sum_{k=1}^{h}(-1)^{k} \frac{V^{k}}{k !}-\sum_{k=2}^{h}\left[3^{k}(3 / 4)^{n / 2}+5^{k} 2^{-n}\right] \frac{V^{k-1}}{(k-1) !}
\end{aligned}
$$

Using the Taylor expansion of $e^{-V}$ with a remainder after $h+1$ terms, we see that this implies that

$$
\begin{aligned}
\int_{F} \alpha\left(\Omega \Lambda_{0}\right) d \mu(\Omega) & \\
& \geqq e^{-V}-\sum_{k=2}^{h}\left[3^{k}(3 / 4)^{n / 2}+5^{k} 2^{-n}\right] \frac{V^{k-1}}{(k-1) !}-\frac{V^{h+1}}{(h+1) !} .
\end{aligned}
$$

If $g$ is even and $g<n$, we obtain, in a similar way

$$
\int_{F} \alpha\left(\Omega \Lambda_{0}\right) d \mu(\Omega) \leqq e^{-V}+\sum_{k=2}^{g}\left[3^{k}(3 / 4)^{n / 2}+5^{k} 2^{-n}\right] \frac{V^{k-1}}{(k-1) !}+\frac{V^{g+1}}{(g+1) !} .
$$

A combination of both these inequalities gives

$$
m(A(S))=\int_{F} \alpha\left(\Omega \Lambda_{0}\right) d \mu(\Omega)=e^{-V}(1-R),
$$

and

$$
\begin{aligned}
& -e^{V}\left[\sum_{k=2}^{o}\left[3^{k}(3 / 4)^{n / 2}+5^{k} 2^{-n}\right] \frac{V^{k-1}}{(k-1) !}+\frac{V^{o+1}}{(g+1) !}\right] \leqq R \\
& \leqq e^{V}\left[\sum_{k=2}^{h}\left[3^{k}(3 / 4)^{n / 2}+5^{k} 2^{-n}\right] \frac{V^{k-1}}{(k-1) !}+\frac{V^{h+1}}{(h+1) !}\right] .
\end{aligned}
$$

But, provided $1 \leqq k \leqq n$, we have

$$
5^{k} 2^{-n}=3^{k}(5 / 3)^{k} 2^{-n}<3^{k}(5 / 6)^{n}<3^{k}(3 / 4)^{n / 2} .
$$

So

$$
\begin{aligned}
& \sum_{k=2}^{h}\left[3^{k}(3 / 4)^{n / 2}+5^{k} 2^{-n}\right] \frac{V^{k-1}}{(k-1) !} e^{V} \\
&<6(3 / 4)^{n / 2} \sum_{k=2}^{h} \frac{3^{k-1} V^{k-1}}{(k-1) !} e^{V}<6(3 / 4)^{n / 2} e^{4 V}
\end{aligned}
$$


Now take $h$ to be odd and to have either the value $n-1$ or the value $n-2$. Then as $V<n-1$ we have

$$
\frac{V^{h+1}}{(h+1) !} e^{V} \leqq \frac{V^{n-1}}{(n-1) !} e^{V} .
$$

Since

$$
e^{n}>n^{n-1} /(n-1) !
$$

it follows that

$$
\frac{V^{h+1}}{(h+1) !} e^{V}<V^{n-1} n^{-n+1} e^{V+n} .
$$

Using (24) and (25) in (22) we obtain

$$
R<6(3 / 4)^{n / 2} e^{4 V}+V^{n-1} n^{-n+1} e^{V+n} .
$$

A similar argument shows that

$$
R>-6(3 / 4)^{n / 2} e^{4 V}-V^{n-1} n^{-n+1} e^{V+n} .
$$

A combination of these inequalities gives (3) and proves Theorem 4.

Theorem 5 (Improvement of the Minkowski-Hlawka Theo$\mathrm{REM})$. Let $S$ be a Borel set, not containing the origin 0. Suppose

$$
V \leqq \frac{1}{8} n \log 4 / 3-\frac{1}{2} \log 3 .
$$

Then there exists an admissible lattice $\Lambda$ with determinant 1.

In the original Minkowski-Hlawka Theorem there is $V<1$ instead of (26). It was first proved by E. Hlawka [1]. In the meantime it was proved to be true for $V<2 /\left(1+2^{1-n}\right)\left(1+3^{1-n}\right)$ by the author [4] and for $V \leqq n^{1 / 2} / 6$ if $n$ is sufficiently large by C. A. Rogers [3].

Proof of Theorem 5. We may assume that $X \in S$ implies $-X \notin S$. We may also assume $n \geqq 13$, because if $n<13$, then (26) yields $V<1$, and the theorem is true. (26) implies (1). Hence (2) and (3) hold. (26) also implies

$$
6(3 / 4)^{n / 2} e^{4 V} \leqq 2 / 3 .
$$

Further, as $\log 4 / 3<1 / 3$, we have $V<n / 24$. Also $e^{25 / 24}<24 / 23$. Thus

$$
\begin{aligned}
V^{n-1} n^{-n+1} e^{V+n} & <(1 / 24)^{n-1} e^{25 n / 24} \\
& <24(24)^{-n}(24 / 23) \\
& =24(23)^{-n}<1 / 3 .
\end{aligned}
$$


Combining these we obtain $|R|<1$, so that $m(A(S))>0$. Consequently, there exists an admissible lattice of determinant 1 .

Theorem 6. Let $S, T$ be two Borcl sets. Assume that $X \in T$ yields $-X \notin S \cup T$ and that $0 \notin S$. Further assume.

$$
\begin{aligned}
V(S) & \leqq \frac{1}{16} n \log 4 / 3-\frac{1}{2} \log 3-4(3 / 4)^{n / 2}, \\
V(S \cup T) & \geqq V(S)+4(3 / 4)^{n / 4} .
\end{aligned}
$$

Then there exists a lattice $\Lambda$ with determinant 1 which is $S$-admissible, but not T-admissible.

Proof of Theorem 6. We may assume that $X \in S$ yields $-X \notin S$. Then never both $X \in S \cup T$ and $-X \in S \cup T$. We introduce $S_{1}=S$, $S_{2}=S \cup T$. We may assume that equality holds in the second equation (27), that is,

$$
V\left(S_{2}\right)=V\left(S_{1}\right)+4(3 / 4)^{n / 4} .
$$

Then

$$
V\left(S_{i}\right) \leqq \frac{1}{16} n \log 4 / 3-\frac{1}{2} \log 3 .
$$

Writing $\alpha_{j}(\Lambda)=\alpha_{S_{j}}(\Lambda), V_{j}=V\left(S_{j}\right), R_{j}=R\left(S_{j}\right), c=(3 / 4)^{n / 4}$, and applying Theorem 4 we infer

$$
\int_{F} \alpha_{i}\left(\Omega \Lambda_{0}\right) d \mu(\Omega)=e^{-V_{i}}\left(1-R_{i}\right)
$$

where

$$
\left|R_{i}\right| \leqq \frac{2}{3}(3 / 4)^{n / 4}+24(23)^{-n} \leqq(3 / 4)^{n / 4}=c<\frac{1}{2} .
$$

Hence

$$
\begin{aligned}
& \int_{F}\left[\alpha_{1}\right.\left.\left(\Omega \Lambda_{0}\right)-\alpha_{2}\left(\Omega \Lambda_{0}\right)\right] d \mu(\Omega)=e^{-V_{1}}\left(1-R_{1}\right)-e^{-V_{2}}\left(1-R_{2}\right) \\
&=e^{-V_{2}}\left[e^{V_{2}-V_{1}}\left(1-R_{1}\right)-\left(1-R_{2}\right)\right] \geqq e^{-V_{2}}\left[e^{4 c}(1-c)-(1+c)\right] \\
&>e^{-V_{2}}[(1+4 c)(1-c)-(1+c)]=e^{-V_{2}}\left(2 c-4 c^{2}\right)>0 .
\end{aligned}
$$

Consequently, there exists a lattice $\Lambda$ satisfying $\alpha_{1}(\Lambda)-\alpha_{2}(\Lambda)>0$. This implies $\alpha_{1}(\Lambda)=1, \alpha_{2}(\Lambda)=0$. Therefore there is a point of $\Lambda$ in $S_{2}=S \cup T$, but no point of $\Lambda$ in $S_{1}=S$. Thus $\Lambda$ is $S$-admissible, but not $T$-admissible. 
TheOREm 7. Let $S_{1}, \cdots, S_{m}$ be $m$ Borel sets in $R_{n}, n \geqq 13$, each so that $X \in S$ yields $-X \notin S$ and with

$$
\sum_{j=1}^{m} e^{-W V_{i}}\left[1+R\left(n, V_{j}\right)\right] \leqq 1,
$$

where $W_{j}=\min \left(V_{j}, n-1\right)$ and $R(n, V)=6(3 / 4)^{n / 2} e^{4 V}+V^{n-1} n^{-n+1} e^{V+n}$. Then there exists a lattice with determinant 1 which has at least one point in each $S_{j}$.

Proof of Theorem 7. Clearly it is enough to prove the theorem if $V_{j} \leqq n-1$. We obtain

$$
\int_{F}\left[\sum_{j=1}^{m} \alpha_{j}\left(\Omega \Lambda_{0}\right)\right] d \mu(\Omega)<\sum_{j=1}^{m} e^{-V_{j}\left[1+R\left(n, V_{j}\right)\right] \leqq 1} .
$$

Consequently, there exists a lattice $\Lambda$ such that $\sum_{j=1}^{m} \alpha_{j}(\Lambda)=0$ and $\Lambda$ is not admissible for any $S_{j}$.

\section{REFERENCES}

1. E. Hlawka, Zur Geometrie der Zahlen, Math. Zeit. vol. 49 (1944) pp. 285-312.

2. C. A. Rogers, Mean values over the space of lattices, Acta Math. vol. 94 (1955) pp. 249-287.

3. - The number of lattice points in a set, Proc. London Math. Soc. vol. 22 (1956) pp. 305-320.

4. W. Schmidt, Eine Verschärfung des Satzes von Minkowski-Hlawka. Monatshefte für Mathematik vol. 60 (1956) pp. 110-113.

5. C. L. Siegel, $A$ mean value theorem in geometry of numbers, Ann. of Math. vol. 46 (1945) pp. $340-347$.

Montana State University 